a journal of music, thought and technology

INSTITUUT

\author{
Title: \\ Subtitle: \\ Author(s): $\quad$ Tom Jackson \\ Issue: \\ ARCHIVE(S) \\ Publication date: $\quad 20^{\text {th }}$ November 2020 \\ Article DOI: $\quad 20^{\text {th }}$ November 2020
}

The musical instrument as archive in free improvisation

\begin{abstract}
This paper discusses ideas surrounding the notion of the "archive" and how they might provide insight into ways musical instruments provide information, inspiration or insights during their use in free improvisation. This is approached through the lens of art theorists and practitioners of free improvisation by consulting texts, interviews and critiques. I offer insight into my own approach to performing in this context and I provide my own solo recordings of clarinet performances that were made with direct reference to the ideas discussed. I also offer a recording of a concert made in a normal performative context.
\end{abstract}

\title{
Introduction
}

"Archives are actually about the future [...] No, they're about the past."

Above is a paraphrase of a discussion regarding the archive of the London Musicians Collective, which was salvaged from skips when their offices had to be cleared after funding cuts. No space could be found for the vast boxes of posters, flyers, programmes, newsletters and minutes of their meetings and events, but at the eleventh hour someone heard about this desperate discard and stepped in to house them.

What good could this archive provide? What interest could be generated from seeing all of these documents from decades ago? Are they about/of (describing) the past or are they about/for (inspiring, informing) the future? Certainly, I was thrilled reading the names of so many people I had met since moving to London in 2009; the same people still striving to progress the music, unperturbed by an activity not without external and internal challenges. It also gave me hope for the future of the music and provided further context of the lives of the people who have been performing free improvisation for decades.

Thinking about archives in this way reminded me of my concerns surrounding the instruments that we use in free improvisation. Are instruments about the past, representing and curating the sounds they were designed to make and have made countless times? Or are they about the future, a collection of unintended possibilities waiting to be discovered? I confront this dichotomy when I am improvising. Is the instrument an archive and who is it for? Is it of the past or for the future? Am I playing it or is it playing me?

In this paper, rather than suggest a fixed definition for how we might think of an archive, I will instead discuss different ways in which the notion of 'archive' could be thought of in the specific context of musical instruments being used in the field of free improvisation.

I begin by setting out a brief account of how tools in general have been theorised, with particular consideration to how they are used and how they provide information to the user, drawing in particular on the work of Jacques Monod. With particular reference to artistic tools, I draw on art theory including writers Vilem Flusser, Ernst Gombrich and James Elkins, and I consider musical instruments along the lines illuminated. I outline some of the ways that practitioners of free improvisation - Pauline Oliveros, Steve Lacy, Derek Bailey, Edwin Prevost, John 
Butcher, Evan Parker and Peter Evans - have discussed their instrument as a text (a collection of instructions), a site of ideas and an influence on the imagination, before setting out some of my own thoughts on the role my instrument, the clarinet, has taken in my improvisational thinking.

With reference to my praxis I offer solo recordings from April 2015 which were recorded at Canterbury Christ Church University with the specific issues discussed in this essay close to mind. For each recording I provide some comments linked to the ideas in the text but rather than concentrating wholly on specific issues I suggest that a residual reflection of the discussion will be sufficient. I end with a link to a recording of a solo performance from a concert at Iklectik, London in April 2018.

In the first recording, I invite the reader to begin to become acquainted with my practical approach and to consider where the ideas and sounds originate. Please refer to recording C1 via https://roamreleases.bandcamp.com/album/tom-jackson-solos which was recorded at St Gregory's Church, Canterbury Christ Church University in April 2015. It was recorded, mixed and mastered by Matt Davison.

\section{Tool use}

Tool use is a consistent part of human experience across cultures and histories. Tools extend our operational potential beyond our immediate physicality, enabling a shift from what is possible with just the use of the body to what is intellectually imaginable and then tangible with the use of external materials.

Jacques Monod suggests a dichotomy between "the natural and the artificial” objects, defining artificial objects as "products of human art or workmanship.” (Monod, 1971, p. 15) For Vilem Flusser a tool is the result of matter being informed: “The object acquires an unnatural, improbable form; it becomes cultural.” (Flusser, 2014, p. 23) In these descriptions the artificial object and the tool have an intended function: they are a physical manifestation of human thought.

Tools are thus testaments to the imagination. From stones halved to reveal sharp bludgeoning weapons to metal spears that leave the human grip (a disembodied flight of human physicality) tools are objects that offer a unique understanding into their user's biological, cultural and chronological context. A tool is an archive of ideas. As Monod writes:

"The object renders in material form the pre-existent intention that gave birth to it, and its form is explained by the performance expected of it even before it takes shape.” (Monod, 1971, p. 15)

Flusser describes the genesis of tools as coming from a desire to enhance our standard biological apparatus; exaggerations of our bodies' natural abilities:

"Tools in the usual sense are extensions of human organs: extended teeth, fingers, hands, arms, legs. As they extend they reach further into the natural world and tear objects from it more powerfully and more quickly than the body could do on its own. They stimulate the organ they are extended from: An arrow simulates the fingers, a hammer the fist, a pick the toe. They are 'empirical'.” (Flusser, 2014, p. 23)

In this second recording I would like to raise the question of how the clarinet and the imagination work together. Does the clarinet satisfy imaginative requirements or does it provoke them? Please refer to recording C2 via https://roamreleases.bandcamp.com/album/tom-jackson-solos which was recorded at St Gregory's Church, Canterbury Christ Church University in April 2015. It was recorded, mixed and mastered by Matt Davison.

\section{Artist tools}

Tools used for art similarly enshrine ideas that are suggestive of a particular artistic practice. Indeed, the tool and the artistic practice could be thought of as interchangeable. As Ernst Gombrich writes: 
"The word 'style', of course, is derived from 'stilus', the writing instrument of the Romans, who would speak of an 'accomplished style' much as later generations spoke of a 'fluent pen’”. (Gomrbich, 2002, p. 8)

To illustrate the connection between art and the tools that are used to create it, Gombrich refers to Ludwig Richter's account of the different stylistic approaches taken by two groups of artists using different mediums to capture the same scene, suggesting that:

"The artist, clearly, can render onl y what his tool and his medium are capable of rendering. His technique restricts his freedom of choice. The features and relationships the pencil picks out will differ from those the brush can indicate ... Sitting in front of his motif, pencil in hand, the artist will ... tend to see his motif in terms of lines, while, brush in hand, he sees it in terms of masses.” (Gomrbich, 2002, p. 56)

Here Gombrich is making a case for tool-led cognition, suggesting that our thinking is influenced by the tools at hand. James Elkins (2000) advances this notion when he describes Claude Monet's perception of the paintbrush as possessing a set of techniques itself, rather than simply responding to its yielder's command. In this way the materiality of the paintbrush is allowed influence; the tool governs what we perceive.

We have seen that Flusser (2014, p. 23) and Monod (1971, p. 15) both suggest that an object's intended function dictates its design - it satisfies certain requirements. In the same way, the designs and developments of musical instruments are made in response to cultural and aesthetic demands. The intention of good instrument design is to assist a performer in the creation of a particular music - the instrument serves as an archive of specific musical potentials.

In thinking of a musical instrument as a carrier of information it becomes more valuable than the materials it is made from, charged with the expertise of the craftsman. To reimagine this in terms of Flusser and Monod, an instrument represents the work done in informing material from natural objects. Yet it goes further - the instrument contains within it the entire possibility of what the instrument is capable of - an exhaustive, abeyant catalogue of an instrument's capacities, designed and accidental.

A musical instrument attends to a desire to disturb vibrations of air in a manner that surpasses our immediate biological resources, intensifying our interactions with the world. Musical instruments dictate their use because of their empirical nature. This is observed by Pauline Oliveros, suggesting a disconnection of body and production when she writes that,

"instruments such as the accordion, harmonica, bandoneon, and concertina in the mid-nineteenth century ... all distance the performer from his or her own breath. The bellows replace the lungs; the fingers that touch buttons and keys replace lips, tongue, and windpipe.” (Oliveros, 2004)

Instruments place distance between the musician and sound, surpassing our immediate biological resources. Like the empirical tools described by Flusser, instruments extend concepts of embodiment and enhance the role of bodily reach. The instrument is a converter of physical movement into sound.

Objects can further be divided into those that are static, retaining their sole function, and those whose usability is malleable. A musical instrument is an example of adaptable functionality; it can inspire new uses. Instruments exist in correlation with a cultural condition but their use is flexible. Improvising with an instrument is an example of humanity's innate interest in exploring the world. Like the metal spear described above, instruments provide aim at otherwise inaccessible ideas. When we use an instrument the ideas it provides render influence on our reach; it is an active archive of influence.

In the third recording I wish to raise the question of how instruments respond to the body and vice versa as well as to consider that their use provokes an adaption in the imaginative line. Please refer to recording C3 via 
https://roamreleases.bandcamp.com/album/tom-jackson-solos which was recorded at St Gregory's Church, Canterbury Christ Church University in April 2015. It was recorded, mixed and mastered by Matt Davison.

\section{Improvisers and Instruments}

By thinking of an instrument in this way, an improviser may consider it as a kind of score. As Steve Lacy commented to Derek Bailey: "The instrument - that's the matter - the stuff - your subject.” (Lacy in Bailey, 1993, p. 99) The special relationship between a musician and their instrument is heightened in free improvisation because of the instant malleability of approaches available to improvisers. For Edwin Prévost: "No musician acts more individually than the improvisor.” (Prévost, 1995, p. 89) and as John Butcher writes, improvisers are invited to undergo a detailed exploration of their instruments:

"A characteristic of much improvisation of the last four or five decades has been the utilization of "new" sound. Musicians and composers in all realms usually have a passion for sound in the abstract, but improvisers have a special, and practical, recognition of how less common sounds lead to new concepts of performance (and vice versa).” (Butcher, 2011)

Butcher is sceptical of the now ubiquitous use of "extended techniques" by composers: "Because they are rarely derived from the player's own needs and personality they invariably end up sounding like the awkward appendages they are.” (ibid.) In contrast, through detailed communion with their instrument, improvisers develop an expertise for refining "less common sounds" as well as a skill for deploying them in unplannable performance contexts. A complexity of sounds expands alongside a player's individual physical investments both in practice and performance. For Butcher the saxophone is a rich source of sonic material both in its intended and accidental characteristics. He writes:

"I've always felt it useful to restrict myself to sonic material rooted in the saxophone's acoustics - to be aware of its traditions but to play with an ear for what lies hidden around the corner. I'm continually engaged with the mechanical and acoustic properties of a tube of metal in my hands and a piece of wood vibrating in my mouth. Even when I work with saxophone controlled feedback (nothing more than a microphone in the instrument's bell connected to an amplifier) it still sounds like a saxophone to me because of the overtone structure embedded in its design and the use of pads and tone holes to change resonances.” (Butcher, ibid.)

The design and constant refinement of an instrument reflects the ideals of the music it has been associated with. Instruments interpret and transform the postural gestures of performers. Hovering over the keys of an instrument, fingers ready themselves for movements as if imagining what might be played, an automatic posture honed by years of practice. Musicians' fingers habitually know how to render chromatic material with such certainty as to make deviation uncomfortable. John Corbett writes:

"If "correct" technique has been formed in conjunction with possible positionings of the performer in relation to the instrument, so has the instrument become complicitous in its very materiality. It is literally composed and manufactured by culture and its possibilities are previously encoded to the degree that the instrument facilitates facility. Implicit in the instrument are techniques for playing it; the knowledge one can have on an instrument is mapped out progressively in terms of a training that allows the musician to move only a certain way and thereby forces the instrument to sound only a certain way.” (Corbett, 1995, p. 229)

In this way instruments are an archive for standardising human action; tools serve as a filter of human interaction and any swerving of this results in a resistance. For Gombrich there is a fusion between the outcome of the product, the 'style', and the tools an artist uses to realise it. When Gombrich says "style rules" (Gombrich, 2002, p. 56) he is suggesting "stilus rules", that we are the servants of the medium we operate. The notion that tools frame what an artist can achieve mirrors Eric Clarke's suggestion that a musical instrument has a grain, a given direction that shapes the potential output. (Clarke, 2010, p. 42) Sonic material is an abstraction from the physical. 
Rather than picking out features differently, using the tools to the best of their ability, Gombrich's language suggests he believes that the faculty of comprehension is altered by the type of tool in hand, that the tool acts to prejudice artistic thought, having a direct impact upon cognition.

While some musicians opt to play against the design of their instrument, to reveal accidental/residual qualities of its physical condition, the instrument remains representative of itself. Gombrich uses the photographer's skill of choosing different exposures and papers to illuminate different possibilities of a negative as a further example of the limiting effect of a medium and moves to a general expression that,

"the artist, too, cannot transcribe what he sees; he can only translate it into the terms of his medium. He, too, is strictly tied to the range of tones which his medium will yield." (Gombrich, 2002, p. 30)

Our imaginations are tied to the physicality of our chosen medium. As Graham Harman suggests, the carrier of art has become art:

"Only in artworks do rock, metal, and color first become what they are rather than being absorbed and suppressed by some ulterior function. Both masons and sculptors use stone. The difference is that the mason uses up the stone by fully assigning it to some practical purpose, while the sculptor lets the stone shine forth as what it is.” (Harman, 2013, pp. 111-2)

This is important for the improvising musician. The performer can work with and against the design of the instrument, but also with the qualities of the matter that is required to fulfil the instrument's design. It is in this light that I invite the reader to listen to the fourth recording, without adding any further prescription. Please refer to recording C1 via https://roamreleases.bandcamp.com/album/tom-jackson-solos which was recorded at St Gregory's Church, Canterbury Christ Church University in April 2015. It was recorded, mixed and mastered by Matt Davison.

\section{Instrumentality and Materiality}

For improvisers then, instrumentality (what it is designed for) and materiality (the innate physical characteristics) are crucial considerations, which we can think of as different archives of the instrument to interrogate. Alex Ward and Xavier Charles are two clarinettists who have reached an advanced sophistication in free improvisation and whose approaches demonstrate the two ways of considering the instrument that I have been describing. Hearing these musicians work with their instruments is a riveting experience and I attempt to describe their work here.

Ward's chromatic, multi-intervallic flourishes are pyrotechnical in their brilliance with a speed of digital movement matched by seemingly endless invention of patterns that develop or disperse at his will. In contrast, Charles magnifies the effect of air rushing through the clarinet's inner column with intricate, delicate and brutal embouchure manipulations colliding with complex fingerings to reveal trembling palettes of the sound of the clarinet's matter.

Rather than perform precisely controlled sounds or patterns, the importance for both Ward and Charles seems to me to be in locating sonic material through performance, guided by the instrument itself. The instrument's design and material construction receive detailed scrutiny from these two virtuosic performers. I do not mean to imply that Ward ignores the materiality of the clarinet, nor that Charles's timbres and textures are void of an awareness of instrumentality, rather that in these two pronounced respects they have demonstrated particular refinement. Through different paths they are both revealing what was always residing in the body of clarinet, archives waiting to be illuminated with breath. 
Attitudes to one's instrument amongst improvisers are certainly not universally agreed. Violinists Bennett Hogg (2011) and Stephen Nachmanovitch (1990) both suggest the instrument amplifies the condition of the human. David Borgo's discussion (2007) of the combined unit of instrument and musician contrasts this position when he comments “in important ways [Evan Parker’s] horn shapes his playing”. Similarly, Derek Bailey spoke of the relationship with his instrument in this way, akin to Gombrich's argument, that improvisers are led by the capabilities of their instruments. The affecting presence of the guitar on the choice and development of elements that Bailey explored, both in practice and performance, has been noted by Dominic Lash (2011) who states,

"while Bailey’s playing aimed at the greatest malleability and adaptability, it was nonetheless subject to a great many constraints which governed its coherence, one of which was the physical construction of the guitar itself.” (Lash, 2011, p. 148)

And Bailey said in 2004:

“I wouldn’t do what I do on any other instrument. It’s very specific. I like the construction of it and the basic tuning, like fourths and a major third. That plays a significant part in what I play, harmonics, open strings, fourths.” (Bailey in Keenan, 2004, p. 44)

Revealed in these two quotes is, for musicians like Bailey, the necessity of a relationship with the instrument as a reliable interface. Bailey cements this position when he writes:

"The instrument is not just a tool but an ally. It is not only a means to an end; it is a source of material, and technique for the improviser is often an exploitation of the natural resources of the instrument.” (Bailey, 1993, p. 99)

Lash elaborates on Derek Bailey’s approach of a clear link between the construction of the guitar and his style:

“[T]he open string was conceptualized by Bailey as one of the three basic timbral varieties of sounds with stable pitch available on the guitar, alongside fretted notes and natural harmonics.” (Lash, 2011, p.145)

The construction of the guitar and its role in producing material for Bailey can be seen in all musical instruments. In their general use, most western instruments work to standardise pitch production within the chromatic scale. This simple example illustrates how an instrument organises the musician's actions - there is a range of positions on a guitar's fretboard or the piano's keys which will result in the same pitch. This is an example of how an instrument quantifies action - the need for precision is diffused over a greater area. If we extend this concept, we can consider an instrument as representing a set of physical parameters to be utilised; a database of possibility, a site where physical memory is ignited. As Peter Evans writes:

"I've started to think of an instrument as many things all at once: a composition, a body of texts, a history, a noise-maker, an amplifier of ideas, and a real extension of the human body.” (Evans, 2009, p. 116)

An instrument simultaneously informs the musician and gives voice to the musician's actions. It is an archive of a complex set of variables that can never be fully mastered. As Evan Parker says:

"You couple yourself to that instrument and it teaches you as much as you tell it what to do. So you're sensitive to ... how it's responding to your efforts to control it. By hearing it, the way it's feeding back to you, you learn to control it better, so it's a very dynamic and very sensitive process . . . [But] the instrument at the same time seems to be giving you additional information so that there are things you have under your control, but every so often something will go wrong. You'll lose control. [And] in that moment you are given an opportunity to learn something else that the instrument can do . . the nature of the instrument and its will in relation to its destiny ... [its] set of intentions in its relationship with you, and you start to find it difficult to distinguish yourself and your intentions from the instrument’s intentions.” (Parker in Hopkins, 2009 - transcribed by Daniel Fischlin, 2009) 
What Parker refers to as "something will go wrong” are instances that reveal a misalignment of instrumental handling and desired sonic result. Rather than being an error, Parker explains that he sees it as an "opportunity to learn something else”. For Parker "ineliminable phenomena” are an instigator of ideas from an undepletable source. Parker embraces the limitations of knowledge that can be achieved as a source of material for investigation in performance, a product of the boundaries of a human/nature relationship. His praxis over the length of his career may have refined this process, but it is this instrumental friction that has continued to guide his approach. Elkins supports this notion in relation to many disciplines when he states:

"Despite the rise of multimedia, film, video, and installation, the majority of artists master their materials, and the majority of painters do not stray any farther toward modern technology than acrylic paints or brushed aluminium: not because they are suspicious of technology, but because there is so much to learn about even the simplest substances.” (Elkins, 2000, p. 34)

An interesting example of this is John Cage's instructions to perform on un-mastered instruments, in particular cacti: "the instruments are so unknown that as you explore, say the spines of a cactus, you're not really dealing with your memory or your taste. You're exploring. As you play you destroy the instrument - or change it because when you make a spine vibrate it begins to lose some of its pliability." (Cage in Feisst 2009, p. 45) This exemplifies Nicholas Cook's description of improvisation as heterarchical, the real-time arrangement of elements that are not pre-defined in temporal terms (Cook 1992, p. 332) and also reminds us of the questions about the role of memory. For Cage’s cacti, memory and the traditional approach to technique are redundant. Instead, the resistance in the spine provides the player with the necessary information each time they start to pluck it. The spine becomes an ever-changing archive of what is currently possible and the necessary technique becomes the player's ability to develop the unknowable.

An elaboration on this notion is provided by Parker when he describes how he works with the saxophone (including circular breathing, top-bottom tonguing and cross-fingering techniques) and explains that this has informed his awareness of what the saxophone represents. He writes:

"All the technical considerations mentioned above are part of a total developing awareness of the instrument as a channel for the imagination but at the same time as a shaper and perhaps limiter of the imagination. In the end the saxophone has been for me a rather specialised bio-feedback instrument for studying and expanding my control over my hearing and the motor mechanics of parts of my skeleto-muscular system and their improved functioning has given me more to think about. Sometimes the body leads the imagination sometimes the imagination leads the body.” (Parker, 1992)

Parker's approach expands the notion of notation suggesting that engagement with the instrument itself can represent the source material (or archive) to which an improviser refers. Comparing this with the example of Cage's cactus, we can see that they both provide the player with something to work with. But where the cactus serves to eliminate the requirement of a particular type of memory, for Parker the saxophone contributes to his memory. Whereas Cage enjoys the imperfectible response to a cactus's spines and disallowance of precise techniques, Parker's approach calls for the instrument's behaviour to invoke practiced responses in new ways. The improviser can think of their instrument as a store of possibilities that seduce known and unknown physical gestures.

Rather than memorise a set of gestures to convey a sense of mastery, the instrument provides and enhances memory as an external store of possibilities that are accessed through playing. Muscle memory, so important to most other performers, is also vital to improvisers, but it must be a malleable memory. It is the training of our bodies to assist instruments in their seduction of human movement through sound.

\section{My use of the clarinet in free improvisation}


The author invites the reader to listen to For Vid Drašler via https://roamreleases.bandcamp.com/album/tomjackson-solos before or while reading this section. This recording presents my own praxis in a concert setting, when the issues discussed in this paper were not at the forefront of my approach but rather provided an implicit environment. This performance was recorded, mixed and mastered in April 2018 by Benedict Taylor at Iklectik Art Centre in London.

It is in light of the closing remarks above that I now examine ways in which I have conceptualised my use of an instrument. As a clarinettist I used to feel at times that I was playing against the design of the instrument, pushing it to go against its physical programming, but I now regard this as exploiting the clarinet's accidental features: multiphonics that rise out of the clarinet's complex handling of the overtone series; bent notes dragged around by the embouchure; immeasurable pitches dancing via subtle sliding of the fingers over tone holes and non-traditional use of the clarinet's keys. There was room to operate outside of the intentions of the clarinet's design and as I continued in this way I cultivated an interest in what its physical condition could sound like.

In my practice of free improvisation the clarinet has become an object of interaction to explore physical and sonic phenomena. Like Cage's cacti spines the clarinet is complex in its consistency and deterioration. The clarinet's body is always degrading, its internal properties morphing with the rush of air and the gradual disintegration of matter. The reed weakens as saliva turns starch into sugar. The clarinet is not a static, stable object, it exists in flux, demanding that the clarinettist adapt and work with minute unknowns that spark huge variations. It is a morphing archive that develops as it reveals, and every revelation is an offering to the ongoing imaginative work necessary for continual development.

When I play, the clarinet's inherent characteristics (both intentional and accidental) serve as source material to issue investigative possibilities. The number of fingerings available multiplied by innumerable tongue positions as well as dynamic approaches present a vast opportunity for exploration. Gaining competence over these techniques is reassuring and rewarding but rather than attempt to create an exhaustive catalogue (which anyway would be impossible) I rely on an incomplete and therefore catalytic understanding of the clarinet's possibilities. In this way I use free improvisation as a vehicle for revelation that is put to work to investigate the clarinet. Some of the best work happens when I get an unexpected result from a physical configuration that I had previously thought of as secure; an alert towards the protean conceptions of the fragile relationship between sound and physicality.

This relationship with an instrument is the crux of my work as an improviser; I am in constant pursuit of ways the clarinet can amplify our conjoined physicality. This involves adopting new gestures and approaches to provide distance from the clarinet's standard use, allowing hidden areas of the clarinet's capacity to come to the fore. I work to uncover the clarinet's archive of materiality in my hands.

Instead of just mastering various techniques, I aim instead to develop an approach to playing that sees all techniques as intrinsic, yet constantly developing and emerging, components. Just as I have no claims to have invented standard approaches, I have no right to call any other approach my own either. What I do possess is the particular combining, ordering and development occurring through performance. The techniques and approaches I use originated because of the investigation into how the clarinet and I work together. If I can lay claim to anything, it is this relationship.

As a cultural object the clarinet came to me as an archive loaded with prejudiced intentions that stretch back through its history, representing various decisions of people and cultures. It also came to me as an object in its own right, as an archive of possible responses without concern for history. It is with regard to these states that I must respond as an improviser.

I have often been sceptical of the role the instrument has on improvisatory thought, concerned that its physical influence would taper sonic ideas into cultural conformity. But thinking of the instrument not only as an archive for amelioration of cultural reproduction but also as an archive of latent physical phenomena has helped to 
relegate the importance of my own ideas to make space for something more important. That's where the idea of an instrumental archive gives me hope.

\section{Bibliography}

Amplified Gesture: An Introduction to Free Improvisation: Practitioners and Their Philosophy (2009) Directed by Phil Hopkins [DVD]. Samadhi Sound.

Bailey, D. (1993) Improvisation: its nature and practice in music. USA: Da Capo Press, Inc.

Borgo, D. (2007) Sync or Swarm: improvising music in a complex age. New York: Continuum Books.

Butcher, J. (2011) Freedom and Sound - This Time it's Personal. Available at:

http://www.pointofdeparture.org/PoD35/PoD35Butcher.html (Accessed: 11 June 2013).

Clarke, E., Dibben, N. \& Pitts, S. (2010) Music and Mind in Everyday Life. Oxford: Oxford University Press.

Cook, N. (1992) Music, Imagination and Culture. Oxford: Oxford University Press.

Corbett, J. (1995) ‘Ephemera Underscored: Writing Around Free Improvisation’, in Gabbard, K. (ed.) Jazz Among the Discourses. London: Duke University Press, pp. 217-238.

Elkins, J. (2000) What Painting Is. New York and London: Routledge.

Evans, P. (2009) ‘Caveman Cyborg’, in Zorn, J. (ed.) Arcana IV: Musicians on Music. New York: Hips Road, Tzadik, pp. 114-120.

Feisst, S. M. (2009) 'John Cage and Improvisation: An Unresolved Relationship’, in Nettl, B. \& Solis, G. (eds.) Musical Improvisation: Art, Education and Society. Urbana: University of Illinois Press, pp. 38-51.

Fischlin, D. (2009) 'Improvisation and the Unnameable: On Being Instrumental', Critical Studies in Improvisation, 5 (1). Available at: http://www.criticalimprov.com/... (Accessed: 20 May 2013).

Flusser, V. (2014) Towards a Philosophy of Photography. London: Reaktion Books.

Gombrich, E. H. (2002) Art and Illusion: A Study in the Psychology of Pictorial Representation. London: Phaidon Press.

Harman, G. (2013) Heidegger Explained: From Phenomenon to Thing. Chicago: Open Court.

Hogg, B. (2011) 'Enactive Consciousness, Intertextuality, and Musical Free Improvisation', in Clarke, D. \& Clarke, E. (eds.) Music and Consciousness: Philosophical, Psychological, and Cultural Perspectives. Great Britain, Oxford University Press, pp. 79-93.

Keenan, D. (2004) 'The Holy Good.’ The Wire, 247, pp. 42-49.

Lash, D. (2011) 'Derek Bailey’s Practice/Practise’, in Boretz, B., Morris, R. \& Rahn, J. (eds.) Perspectives of New Music, 49 (1). Washington: Perspectives of New Music, pp. 143-171.

Monod, J. (1971) Chance and Necessity: An Essay on the Natural Philosophy of Modern Biology. New York: Alfred A. Knopf.

Nachmanovitch, S. (1990) Free Play: Improvisation in Life and Art. New York: Penguin Putnam Inc.

Oliveros, P. (2004) 'Tripping On Wires: The Wireless Body: Who is Improvising?', Critical Studies in Improvisation, 1 (1). Available at: http://www.criticalimprov.com/... (Accessed: 20 January 2013). 
reilo

a journal of music, thought and technology
The musical instrument as archive in free improvisation Dr Tom Jackson

Parker, E. (1992) “De Motu” for Buschi Niebergall. Available at:

http://www.efi.group.shef.ac.uk/fulltext/demotu.html (Accessed: 20 May 2014).

Prevost, E. (1995) No Sound is Innocent: AMM and the Practice of Self-invention. Essex: Copula. 